\title{
Aggressive surgical treatment of multidrug-resistant tuberculosis
}

\author{
Yuji Shiraishi, MD, Naoya Katsuragi, MD, Hidefumi Kita, MD, Yoshiaki Tominaga, MD, \\ Kota Kariatsumari, MD, and Takato Onda, MD
}

\begin{abstract}
Objective: Because extensively drug-resistant tuberculosis has emerged, adequate control of drug-resistant tuberculosis has become increasingly important. We report on our experience using liberal adjuvant resectional surgery as part of aggressive treatment for multidrug-resistant tuberculosis.
\end{abstract}

\begin{abstract}
Methods: We retrospectively reviewed the records of 56 consecutive patients who underwent pulmonary resections for multidrug-resistant tuberculosis between January 2000 and June 2007. There were 42 males and 14 females (mean age, 46 years; range, 22-64 years). Isolates were resistant to a mean of 5.6 drugs (range, 2-10 drugs). Multi-drug regimens employing 3 to 7 drugs (mean, 4.6 drugs) were initiated in all patients. Indications for surgery were a high risk of relapse for 37 patients, persistent positive sputum for 18, and 1 with associated empyema.

Results: The 56 patients underwent 61 pulmonary resections ( 3 completion pneumonectomies, 19 pneumonectomies, 33 lobectomies, and 6 segmentectomies). Bronchial stumps were reinforced with muscle flaps in 54 resections. Operative mortality and morbidity rates were $0 \%$ and $16 \%$, respectively. All patients attained postoperative sputum-negative status. Relapse occurred in 5 patients; 3 were converted by a second resection, and 1 responded to augmentation of chemotherapy. Late death occurred for 2 patients without evidence of relapse. Among 54 survivors, 53 (98\%) were considered cured.

Conclusion: Surgical treatment that complements medical treatment has proved safe and efficacious for patients with multidrug-resistant tuberculosis. In an era with extensively drug-resistant tuberculosis, an aggressive treatment approach to multidrug-resistant tuberculosis continues to be justified until a panacea for this refractory disease is available.
\end{abstract}

The emergence of multidrug-resistant tuberculosis—defined as resistance to at least isoniazid and rifampicin ${ }^{1}-$ has adversely affected the global battle against tuberculosis. ${ }^{2}$ Now that the existence of extensively drug-resistant tuberculosis, a very serious form of multidrug-resistant tuberculosis, has been recognized, ${ }^{3}$ adequate control of drug-resistant tuberculosis is becoming increasingly important. Multidrug-resistant tuberculosis has disseminated world-wide, and Japan is no exception. ${ }^{4}$ As one of the specialized centers for multidrug-resistant tuberculosis in Japan, our hospital has received a number of referrals for treatment of patients with multidrug-resistant tuberculosis. To eliminate a risk of further treatment failure, we have been treating these patients in an aggressive manner. We have performed resectional surgeries for patients, whenever feasible, in order to enhance the efficacy of their medical treatments. Surgical treatment has been used not only to achieve sputum conver-

From the Section of Chest Surgery, Fukujuji Hospital, Tokyo, Japan.

Read at the Eighty-Ninth Annual Meeting of The American Association for Thoracic Surgery, Boston, Mass, May 9-13, 2009.

Received for publication April 7, 2009; revisions received June 3, 2009; accepted for publication July 5, 2009.

Address for reprints: Yuji Shiraishi, MD, Section of Chest Surgery, Fukujuji Hospital, 3-1-24 Matsuyama, Kiyose, Tokyo, 204-8522 Japan (E-mail: yujishi@mvb. biglobe.ne.jp).

J Thorac Cardiovasc Surg 2009; 138:1180-4

$0022-5223 / \$ 36.00$

Copyright (c) 2009 by The American Association for Thoracic Surgery doi:10.1016/j.jtcvs.2009.07.018 sion, but also to prevent a relapse. In this study, we report on our most recent experience with aggressive treatments of patients with multidrug-resistant tuberculosis, with an emphasis on continued liberal use of adjuvant resectional surgery and on reduction of postoperative complications.

\section{PATIENTS AND METHODS}

We retrospectively reviewed the records of 56 consecutive patients who underwent pulmonary resections for multidrug-resistant tuberculosis at Fukujuji Hospital between January 2000 and June 2007. The study was approved, with a waiver for patient consent, by the institutional review board on human research at Fukujuji Hospital.

There were 42 men $(75 \%)$ and 14 women ( $25 \%$ ), including 8 foreigners: 4 Chinese, 2 Filipinos, 1 Nepalese, and 1 Nigerian. Ages at the time of surgery ranged from 22 to 64 years (mean, 46 years). None of the patients was HIV-positive. The average body mass index at the time of admission was 21.0 (range, 14.4 to 29.2). Seventeen patients had diabetes. All but 3 patients had received anti-tuberculosis chemotherapy at their referral hospitals, and had been transferred to our hospital to seek specialized care for multidrugresistant tuberculosis. Sputum smears and sputum cultures were performed upon admission. Drug susceptibility testing was routinely performed on positive cultures in our laboratory, not only for first-line drugs, but also for second-line drugs and levofloxacin. Bronchoscopy was done for some cases to rule out contralateral disease and/or co-existing malignancies.

At the time of admission, patients' isolates were resistant to a mean of 5.6 drugs (range, 2 to 10 drugs), including isoniazid and rifampicin. Multidrug regimens employing a mean of 4.6 drugs (range, 3 to 7 drugs) were initiated in all patients. Treatment regimens were individualized based upon drug susceptibility test results, and comprised as many effective drugs as possible to prevent multidrug-resistant bacilli from acquiring further drug resistance. Fluoroquinolones were included in the regimens of 49 patients $(88 \%)$. Levofloxacin, gatifloxacin, sparfloxacin and ciprofloxacin were used for 26 
patients, 17 patients, 5 patients and 1 patient, respectively. Other drugs employed included ethambutol, streptomycin, kanamycin, enviomycin, cycloserine, ethionamide, para-aminosalicylic acid, pyrazinamide, sultamicillin tosilate and thioacetazone.

After keeping patients under chemotherapy for at least 3 months, we reviewed their sputum smear and culture results and radiographic appearances. Resection of cavitary lesions or destroyed lobes was considered for those patients who had persistently positive sputum, despite chemotherapy. Among patients who achieved sputum conversion in response to chemotherapy, surgery was considered for those at high risk of relapse based upon drug resistance patterns and/or radiographic findings, ie, patients with a higher number of resistant drugs, the bigger size of cavity, and diabetes. Preoperative studies included a chest roentgenogram, computed tomographic scan of the chest, pulmonary function tests, arterial blood gas analysis, and a quantitative perfusion scan. Emphasis was placed upon ascertaining if patients had sufficient pulmonary reserve to tolerate pulmonary resection and if they had lesions that were predominantly localized to one lung. We accepted scattered nodular lesions or limited cavitary lesions in the lungs on the opposite side on the presumption that those lesions could be managed by postoperative chemotherapy or staged bilateral resections.

Radiographic appearances of responsible lesions were cavities in 52 patients, nodular lesions in 2, and a destroyed lobe and empyema in 1 each. Fifteen patients $(27 \%)$ had their tuberculosis limited entirely to 1 side, with the opposite side totally intact. Preoperative vital capacity ranged from 1.6 to $5.78 \mathrm{~L}$ (mean, $3.29 \mathrm{~L}$ ) and forced expiratory volume in 1 second ranged from 0.62 to $4.56 \mathrm{~L}$ (mean, 2.56L). Indications for surgery included persistent positive sputum for 18 patients $(32 \%)$ and a high risk of relapse for 37 patients $(66 \%)$. For 1 patient $(2 \%)$, surgery was performed because he had an associated empyema.

Surgery was performed using the technique previously reported. ${ }^{5}$ We usually performed a posterolateral thoracotomy. Late in this series, we used an anterolateral thoracotomy for selected patients in whom dense pleural adhesions were not anticipated. The bronchus was divided and closed with staples $(n=57)$ or sutures $(n=4)$. We routinely reinforced the bronchial stump with muscle flaps, except when we did segmentectomy. Our preferred muscle was a latissimus dorsi. When the large residual space was considerable, we employed limited thoracoplasty or a latissimus dorsi muscle tent ${ }^{6}$ to reduce the space. All resected specimens were sent for pathological and bacteriological examinations.

After surgery, all patients were maintained on multidrug regimens, generally the same as their preoperative regimens, for 2 years after the time of surgery or after the time of sputum conversion. Durations of postoperative chemotherapy ranged from 6 to 84 months (median, 18 months). Follow-up data were obtained from outpatient or hospital charts, or by direct contacts with patients or relatives. Postsurgical follow-up was completed on December 31, 2008. Durations of follow-up ranged from 8 to 105 months (median, 39 months). Operative mortality included all deaths that were clearly related to the operations, regardless of the postoperative interval. All cases of bronchopleural fistula or empyema that occurred subsequent to the date of surgery were considered postoperative complications.

\section{RESULTS}

Fifty-six patients underwent 61 pulmonary resections. Five patients had 2 resections, 2 had planned staged bilateral resections for bilateral lesions, and 3 had a second resection due to relapse. The procedures included completion pneumonectomy $(\mathrm{n}=3)$, pneumonectomy $(\mathrm{n}=19)$, bilobectomy $(\mathrm{n}=1)$, lobectomy $(\mathrm{n}=32)$, and segmentectomy $(\mathrm{n}=6)$. Surgical procedures were equally divided between the right and left sides (Table 1). Operating times ranged from 120 to 635 minutes (mean, 293 minutes). The average intraoperative blood loss was $297 \mathrm{~mL}$ (range, 10 to $1656 \mathrm{~mL}$ ). Bron-
TABLE 1. Types of pulmonary resections in this series: Early (20002002) and late (2003-2007)

\begin{tabular}{lcc}
\hline \multicolumn{1}{c}{ Procedure } & $\mathbf{2 0 0 0 - 2 0 0 2}$ & $\mathbf{2 0 0 3 - 2 0 0 7}$ \\
\hline Right completion pneumonectomy & 1 & $1(1 \dagger)$ \\
Right pneumonectomy & 5 & 1 \\
Right upper and middle lobectomy & 1 & 0 \\
Right upper lobectomy with segmentectomy* & $4(1 \dagger)$ & $3(1 \dagger, 1 \ddagger)$ \\
Right upper lobectomy & 5 & $7(1 \dagger)$ \\
Right segmentectomy & 1 & 0 \\
Left completion pneumonectomy & 0 & $1(1 \dagger)$ \\
Left pneumonectomy & 6 & 7 \\
Left upper lobectomy with segmentectomy* & 1 & 0 \\
Left upper lobectomy & 5 & $6(1 \dagger)$ \\
Left lower lobectomy & $1(1 \dagger)$ & 0 \\
Left segmentectomy & 3 & $2(1 \dagger)$ \\
Total & $33(2 \dagger)$ & $28(6 \dagger, 1 \ddagger)$ \\
\hline
\end{tabular}

*Upper lobectomy with resection of superior segment of the lower lobe; The numbers of resections $\dagger$ with thoracoplasty or $\ddagger$ with muscle tent are shown in parentheses.

chial stumps were reinforced with muscle flaps in 54 resections. A latissimus dorsi muscle was used in 47 resections, an intercostal muscle in 5 , and other extrathoracic muscles in 2. A concomitant limited thoracoplasty was performed in 8 resections and the latissimus dorsi muscle tent was used in 1 resection. For 10 of the 37 patients $(27 \%)$ who underwent resections for the prevention of relapse, cultures of the contents of their resected specimens yielded positive results.

There was no operative mortality. A total of 11 complications occurred in 10 patients $(16 \%)$ (Table 2). Nine of these 10 patients had been operated on during the first 3 years of this series. In the following $4 \frac{1}{2}$ years, only an occult bronchopleural fistula occurred in 1 patient. This fistula was a complication of a right completion pneumonectomy with a pectoralis major muscle flap and thoracoplasty. It was not associated with clinical symptoms, and it was successfully treated by chest tube thoracostomy.

All patients attained sputum-negative status after their surgeries. Relapse of multidrug-resistant tuberculosis occurred in 5 patients. Three were converted by a second resection, 1 responded to augmentation of chemotherapy and 1 remained positive. The first patient had a relapse around the bronchial stump 21 months after a left upper lobectomy, which resulted in a bronchopleural fistula with tuberculous

TABLE 2. Postoperative complications in this series: Early (20002002) and late (2003-2007)

\begin{tabular}{lcc}
\hline \multicolumn{1}{c}{ Postoperative complications } & $\begin{array}{c}\mathbf{2 0 0 0 - 2 0 0 2} \\
(\mathbf{n}=\mathbf{3 3})\end{array}$ & $\begin{array}{c}\mathbf{2 0 0 3 - 2 0 0 7} \\
(\mathbf{n}=\mathbf{2 8})\end{array}$ \\
\hline Bronchopleural fistula with empyema & 2 & 0 \\
Bronchopleural fistula (occult) & 0 & 1 \\
Space problem & 5 & 0 \\
Prolonged air leak & 2 & 0 \\
Chylothorax & 1 & 0 \\
Total & 10 & 1 \\
\hline
\end{tabular}


empyema. He underwent an open window thoracostomy followed by completion pneumonectomy. The second patient had a relapse in the postlobectomy space 3 months after a right upper lobectomy with segmentectomy. He was treated by completion pneumonectomy following open drainage. The third patient, status post right upper lobectomy with segmentectomy 14 months prior, had a relapse of cavities in the left lung. Because he had had his postoperative chemotherapy terminated 2 months before that, he was returned to a multidrug regimen and underwent a left upper lobectomy. The fourth patient resumed having positive sputum 4 months after surgery while he was under postoperative chemotherapy. He was successfully treated by augmentation of a multidrug regimen. The fifth patient had a relapse around the bronchial stump 5 months after a right pneumonectomy. The bronchial stump was eroding, which resulted in a bronchopleural fistula with tuberculous empyema. He underwent an open window thoracostomy, but the left lung was contaminated with multidrug-resistant tuberculosis. $\mathrm{He}$ is the only patient who remains shedding positive sputum and is under chemotherapy. Late death occurred for 2 patients without evidence of relapse. Among 54 survivors, $53(98 \%)$ were considered cured.

\section{DISCUSSION}

Over the past 3 decades, the presence of multidrug-resistant tuberculosis has been 1 of the major obstacles for the global eradication of tuberculosis. ${ }^{2}$ To improve the efficacy of medical treatment for multidrug-resistant strains, such innovations as fluoroquinolone-containing regimens have been adopted. ${ }^{7,8}$ Recently, the world has entered an era of extensively drug-resistant tuberculosis, ie, multidrug-resistant strains that have acquired additional drug resistance to any fluoroquinolone and to at least 1 of the 3 injectable drugs: capreomycin, kanamycin, and amikacin. ${ }^{3}$ The emergence of extensively drug-resistant tuberculosis may be the result of years of inadequate management of drug-resistant tuberculosis. In order to prevent creating another extensively drug-resistant strain, it is of upmost importance that we cure patients while they have ordinary multidrug-resistant strains.

It is indisputable that the first-line treatment for multidrugresistant tuberculosis is medical treatment. The overall success rate for multidrug-resistant tuberculosis treatment has reached $62 \%$ based on a meta analysis of a variety of current treatment regimens. ${ }^{9}$ However, much higher cure rates have been achieved when a combination of medical and surgical treatments is applied. ${ }^{10-14}$ In our study, the cure rate of $98 \%$ with relatively long-term follow-up is fairly good compared with results of other studies. ${ }^{10-14}$ Our surgically-treated patients comprised 2 types of patients: (1) patients who continued to have positive sputum despite chemotherapy and (2) patients who had sputum converted, but were considered at high risk of relapse. It is unquestionable that the primary goal of adjuvant resectional surgery is to achieve sputum conversion for persistent sputum-positive patients. On the other hand, since medical treatment of multidrug-resistant tuberculosis has been making steady progress, and because surgical treatment has been inherently associated with morbidity, it is debatable whether or not resectional surgery should be utilized liberally for the purpose of preventing relapse in sputum-converted patients.

Since we commenced the use of fluoroquinolone-containing regimens, the efficacy of medical treatment in our program has improved. In this study, $66 \%$ of the patients were sputum-converted prior to surgery, primarily by using levofloxacin- or gatifloxacin-containing regimens. These results are consistent with those in recently reported series. ${ }^{12-14}$ Sputum conversion, however, does not mean that patients are free from active strains, especially when chemotherapy has not been able to transform cavitary lesions into nodular types. Bacteriologic examinations of our resected specimens demonstrated that $27 \%$ of the preoperatively culture-negative patients were still harboring viable bacilli in their target lesions. Therefore, with a view to eliminating the potential risk of relapse, we think that resectional surgery continues to be warranted for patients who have achieved sputum conversion, but who remain with cavities or destroyed lungs.

Without achieving acceptably low operative morbidity and mortality rates, we cannot justify the liberal use of resectional surgery. In this study, the operative mortality was zero, which was consistent with what has been reported in other series. ${ }^{10-14}$ The morbidity rate was $16 \%$, which was a mediocre result, but comparable to the rates in other studies. ${ }^{10-14}$ In our previous study, bronchopleural fistulas and space problems were noticeable postoperative problems. ${ }^{5}$ As we gained more experience, we could prevent these complications late in this series.

Concerning bronchopleural fistulas, the fistulas that we encountered early in this series occurred late after surgery and were associated with relapse. This fact confirmed to us that postoperative chemotherapy should be vigorous enough to control any remaining bacilli around the bronchial stump. We continued to put a muscle flap on the bronchial stump and to employ individualized multi-drug regimens throughout the treatment period. We did not experience a clinically evident bronchopleural fistula during the last $41 / 2$ years of this series.

In addition, space problems became preventable late in this series. In our previous study, the use of a latissimus dorsi muscle flap could not eliminate a risk of space problems. These problems occurred mainly in patients who underwent procedures that were more extensive than lobectomy, such as bilobectomy or lobectomy with segmentectomy. The remaining lobes were inherently too small and too stiff to fill the entire pleural space. For such cases, we found that we had left the raw surface of the remaining lobes open to the post-resectional space when we sewed the latissimus dorsi 
onto the bronchial stump only. Therefore, we modified our technique for dealing with the residual space, either by obliterating the space with concomitant limited thoracoplasty or by covering the raw surface with the latissimus dorsi (muscle tent). ${ }^{6}$ During the last $4 \frac{1}{2}$ years of this series, we did not encounter space problems in any patients.

Postoperative chemotherapy is as indispensable as preoperative chemotherapy. The purpose of resectional surgery is to remove the main lesions that harbor numerous organisms. This means that scattered nodular lesions and tiny cavities may be left behind. In this study, approximately $3 / 4$ of patients had some degree of lesions in the lungs on the opposite side. Hence, it is crucial to keep patients on multidrug regimens for a sufficiently long period in order to hold the bacilli at bay in these lesions. Our duration of postoperative chemotherapy is 2 years, either when patients have positive sputum at the time of surgery or when they have positive specimen culture results. We may shorten the duration of chemotherapy, provided that patients have achieved sputum conversion prior to surgery and have negative specimen culture results. However, the optimal duration of postoperative chemotherapy requires further investigation.

Our study includes several distinctive features. First, as we are affiliated with the Japan Anti-Tuberculosis Association, our institution is one of the most experienced centers for the treatment of multidrug-resistant tuberculosis in Japan. We have such a reliable laboratory that we can routinely perform drug susceptibility testing on first- and secondline drugs and levofloxacin. As we have all types of antituberculosis drugs on hand, we can create the best available multi-drug regimens that are individualized for each patient. Nevertheless, favorable results with adjuvant resectional surgery have been reported not only from developed countries, but also from developing countries where medical resources are limited. ${ }^{13,14}$

Second, we preferred to use gatifloxacin late in this series in expectation of its potent efficacy against tuberculosis bacilli. ${ }^{15}$ Gatifloxacin has, however, been currently withdrawn from sale both in the United States and in Japan because of the increased risk of dysglycemia in humans. This necessitates alternate, newer fluoroquinolones. As we live in an era in which extensively drug-resistant tuberculosis exists, multidrug-resistant tuberculosis should be controlled by any means. An aggressive treatment approach to multidrug-resistant tuberculosis continues to be justified until a panacea for this refractory disease is available.

\section{References}

1. Iseman MD. Treatment of multidrug-resistant tuberculosis. New Engl J Med. 1993;329:784-91.

2. Nachega JB, Chaisson RE. Tuberculosis drug resistance. A global threat. Clin Infect Dis. 2003;(36(Suppl 1)):S24-30.

3. World Health Organization. Extensively drug-resistant tuberculosis (XDR-TB): recommendations for prevention and control. Wkly Epidemiol Rec. 2006;81: 430-2.
4. Tuberculosis Research Committee (Ryoken). Drug-resistant Mycobacterium tuberculosis in Japan: a nationwide survey, 2002. Int J Tuberc Lung Dis. 2007; 11:1129-35

5. Shiraishi Y, Nakajima Y, Katsuragi N, Kurai M, Takahashi N. Resectional surgery combined with chemotherapy remains the treatment of choice for multidrug-resistant tuberculosis. J Thorac Cardiovasc Surg. 2004;128:523-8.

6. Rocco G. Pleural partition with intrathoracic muscle transposition (muscle tent) to manage residual spaces after subtotal pulmonary resections. Ann Thorac Surg. 2004;78:e74-6.

7. Tsukamura M, Nakamura E, Yoshii S, Amano H. Therapeutic effect of a new antibacterial substance ofloxacin (DL8280) on pulmonary tuberculosis. Am Rev Re spir Dis. 1985;131:352-6.

8. Yew WW, Chan CK, Leung CC, Chau CH, Tam CM, Wong PC, et al. Comparative roles of levofloxacin and ofloxacin in the treatment of multidrug-resistant tuberculosis. Preliminary results of a retrospective study from Hong Kong. Chest. 2003; 124:1476-81

9. Orenstein EW, Basu S, Shah NS, Andrews JR, Friedland GH, Moll AP, et al. Treatment outcomes among patients with multidrug-resistant tuberculosis: systematic review and meta-analysis. Lancet Infect Dis. 2009;9:153-61.

10. Sung S-W, Kang CH, Kim YT, Han SK, Shim Y-S, Kim JH. Surgery increased the chance of cure in multi-drug resistant pulmonary tuberculosis. Eur J Cardiothoracic Surg. 1999;16:187-93.

11. Pomerantz BJ, Cleveland JC Jr, Olson HK, Pomerantz M. Pulmonary resection for multi-drug resistant tuberculosis. J Thorac Cardiovasc Surg. 2001;121:448-53.

12. Chan ED, Laurel V, Strand MJ, Chan JF, Huynh MN, Goble M, et al. Treatment and outcome analysis of 205 patients with multidrug-resistant tuberculosis. Am J Respir Crit Care Med. 2004;169:1103-9.

13. Kir A, Inci I, Torun T, Atasalihi A, Tahaoglu K. Adjuvant resectional surgery improves cure rates in multidrug-resistant tuberculosis. J Thorac Cardiovasc Surg. 2006;131:693-6.

14. Mohsen T, Abou Zeid A, Haj-Yahia S. Lobectomy or pneumonectomy for multidrug-resistant pulmonary tuberculosis can be performed with acceptable morbidity and mortality: A seven-year review of a single institution's experience. $J$ Thorac Cardiovasc Surg. 2007;134:194-8.

15. Alvirez-Freites EJ, Carter JL, Cynamon MH. In vitro and in vivo activities of gatifloxacin against Mycobacterium tuberculosis. Antimicrob Agents Chemother. 2002;46:1022-5

\section{Discussion}

Dr Alain Chapelier (Suresnes, France). Dr Shiraishi and colleagues reported their updated experience of patients with multidrug-resistant Mycobacterium tuberculosis (MDR-TB) who underwent adjuvant resectional surgery with no mortality and excellent outcome. Dr Shiraishi has to be congratulated for these results in a technically challenging group of patients and for his nice presentation.

Surgery was considered for the patients with persistent positive sputum and the patients with a high risk of relapse because of residual cavitary lesions. Among this latter group, a positive bacterial examination of specimen was observed in approximately one third of patients who were preoperatively culture-negative, and this is a strong argument for surgery.

In this series, resections were performed with extended use of the latissimus dorsi muscle flap to reinforce the bronchial stump, as it has been also recommended by others. You outline a significant reduction of postoperative complications in the recent period of your experience. No residual pleural space problem was observed in the late period of your study.

I have 3 questions. My first question is related to the additional thoracoplasty. In the situation of a large post-resection space after an upper lobectomy, extended or not to the upper segment of the lower lobe, would you now advocate it systematically?

Dr Shiraishi (Tokyo, Japan). Yes, I would. In our series, we found that space problems often occurred after we performed 
a bilobectomy or lobectomy plus segmentectomy of the lower lobe. To prevent the residual space, we have to close the residual space by concomitant limited thoracoplasty or a muscle tent. I think the important thing is that at first we put the latissimus dorsi just onto the bronchial stump, but in that case we found that the raw surface of the remaining lung was exposed to the pleural cavity and might cause a minor leak and result in residual space. So I think we have to remove some of the ribs to reduce the space and cover the raw surface of the lung with muscle flap.

Dr Chapelier. My second question is concerning the patients with bilateral lesions. Many of your patients had small nodular lesions in the contralateral lung at the time of preoperative assessment. In 2 patients, however, you performed a planned staged bilateral resection. Could you comment on your strategy for these patients?

Dr Shiraishi. The best candidate for surgical therapy was the patient who had limited lesions, for example, a cavity just in the right upper lobe or left upper lobe, but some patients had cavities in both lungs. In that case, if the patient had good pulmonary function and a cavity was just localized in both sides of the upper lobes, we were able to remove both lesions, so we performed planned staged bilateral resections. Also, if the cavity is very small (eg, 5 or $6 \mathrm{~mm}$ ), maybe we can leave those lesions.

Dr Chapelier. Finally, your institution is clearly a referral center for the treatment of MDR-TB in Japan, and you point out in the article the extreme importance of your laboratory to assess the efficacy of all drugs preoperatively and postoperatively. Considering the incidence of MDR-TB in the population of west European countries, for example, the prevalence of newly diagnosed cases is $1.4 \%$ in France with a stability during the last 10 years, and with regard to the medical and surgical expertise required to reach the results you reported today, would you recommend that this combined treatment be done in only 1 or 2 centers in our countries?

Dr Shiraishi. I don't understand your question.

Dr Chapelier. Do you think these difficult cases have to be done in selected centers?

Dr Shiraishi. Oh, yes. Surgery should be done in very specialized and highly experienced centers.

Dr Chapelier. Thank you.

Dr Marvin Pomerantz (Aurora, Colo). I enjoyed your article. Your results parallel our series published in the Journal of 180 resections in 174 patients, which was in 2001. I have 2 questions. Two of 3 of your pneumonectomies were on the left side. Every series I have ever read shows that if you do a pneumonectomy for tuberculosis, it is more often on the left side. Why is that?

Dr Shiraishi. Some groups have termed it "left lung syndrome" in such patients with tuberculosis. The left lung has some anatomic characteristics. The left main bronchus is longer than the right main bronchus. That means more vulnerability to tuberculosis bacilli. So that's why a left-sided pneumonectomy is more often done than a right-sided pneumonectomy.

Dr Pomerantz. Well, I'm not sure that's right. I'm not sure what the answer is either, but it's possibly due to the upper and lower lobe bronchial takeoff being so close together. That's another option. No one has been able to give me a good explanation. You have a higher number of segmental resections than we had in our series. I always thought it was better to make a wider resection than just a segmental. We had several, but not the higher percentages you have.

Dr Shiraishi. We performed a segmentectomy only in patients with very tiny cavities or tiny nodular lesions. We usually do lobectomy because the cavity is bigger. So in our series, the patients who had a segmentectomy had very limited lesions.

Dr Pomerantz. My final question is, you had 2 bronchopleural fistulas. Were they in patients who had positive sputum at the time of surgery, and were they on the right side or the left?

Dr Shiraishi. If I recall correctly, one bronchopleural fistula occurred on the right side after pneumonectomy. The other bronchopleural fistula occurred after left upper lobectomy. Both patients had positive sputum at the time of surgery and comorbidities, such as diabetes. Those patients had bronchopleural fistula 1 or 2 years after surgery, and the bronchial stump was eroded by the relapse of multidrug-resistant strains, and that caused bronchopleural fistula.

Dr Nasser Altorki (New York, NY). I want to ask you a little bit about the segmentectomy. Are you advocating that patients with MDR-TB have a lobectomy even if anatomically a segment can be performed?

Dr Shiraishi. It depends on the patient's pulmonary functional reserve.

Dr Altorki. Assuming that the pulmonary examination allows you to do either of these operations, is a segmentectomy sufficient if you can get rid of gross disease, or do you have microscopic disease that requires a lobectomy be preferred?

Dr Shiraishi. We performed segmentectomy mostly on the left upper division or lingular. In patients with limited lesions, we can take whole lesions by performing a segmentectomy, not a lobectomy.

Dr Altorki. I ask because the seminal work by Churchill and Belsey described segmentectomy exactly in patients with tuberculosis, and I wonder if the fact that they have drug-resistant tuberculosis changes the treatment at all.

Dr Shiraishi. No, it won't.

Dr Altorki. Thank you for your answer. 